\title{
GAIN-SCHEDULED CONTROLLER DESIGN: VARIABLE WEIGHTING APPROACH
}

\author{
Adrian Ilka — Vojtech Veselý ${ }^{*}$
}

\begin{abstract}
Among the most popular approaches to non-linear control is gain-scheduled (GS) controller, which can have better performance than robust and other ones. Our approach is based on a consideration that in linear parameter varying (LPV) system, scheduling parameters and their derivatives with respect to time are supposed to lie in a priori given hyper rectangles. To access the performance quality a new quadratic cost function is used, where weighting matrices are time varying depends on scheduled parameter. The class of control structure includes decentralised fixed order output feedbacks like PID controller. Numerical examples illustrate the effectiveness of the proposed approach.
\end{abstract}

K e y w ords: gain-scheduled control, decentralised control, Lyapunov function, quadratic cost function, MIMO LPV systems, PID controller

\section{INTRODUCTION}

Consider a linear parameter varying (LPV) system with state space matrices which are fixed functions of known vector parameter varying $\theta(t)$. This model can be a linear time invariant (LTI) plant model which is result from linearisation of the non-linear plants along trajectories of the known parameter $\theta(t) \in\langle\underline{\theta}, \bar{\theta}\rangle$. In this note the following LPV system will be used

$$
\begin{aligned}
& \dot{x}=A(\theta(t)) x+B(\theta(t)) u \\
& y=C x
\end{aligned}
$$

where

$$
\begin{aligned}
& A(\theta(t))=A_{0}+A_{1} \theta_{1}(t)+\ldots+A_{p} \theta_{p}(t) \\
& B(\theta(t))=B_{0}+B_{1} \theta_{1}(t)+\ldots+B_{p} \theta_{p}(t)
\end{aligned}
$$

and $x \in R^{n}$ is the state, $u \in R^{m}$ is a control input, $y=R^{l}$ is the measurement output vector, $A_{0}, B_{0}, A_{i}, B_{i}$, $i=1,2 \ldots p, C$ are constant matrices of appropriate dimension, $\theta(t) \in\langle\underline{\theta}, \bar{\theta}\rangle \in \Omega$ vector of time-varying plant parameters.

The main motivation for our work lies in [1-5]. In [1] the author tackles the design problem of gain scheduled controllers for LPV systems via parameter-dependent Lyapunov function. Recently, [2] proposed the design method for gain scheduled problem using a similar technique to [1]. Improved stability analysis and gain scheduled controller synthesis for parameter-dependent systems are proposed in [3]. Survey of scheduled controller analysis and synthesis are presented in $[4,5]$.

In this note our approach is based on

- A consideration of the LPV systems (1), scheduling parameters $\theta_{i}, i=1,2, \ldots, p$ and their derivatives with respect to time are supposed to lie in a priori given hyper rectangles, $\theta \in \Omega$ and $\dot{\theta} \in \Omega_{t}$.

- Affine quadratic stability (AQS) introduced by [6].

- We use the notion of guaranteed cost to guarantee the performance of closed-loop system.

- The class of control structure includes decentralised fixed order output feedback like PID controller.

The paper is organised as follows. Section 2 brings preliminaries and problem formulation. The main result is presented in Section 3. In Section 4, numerical example illustrate the effectiveness of the proposed approach.

\section{PRELIMINARIES AND PROBLEM FORMULATION}

Consider an LPV system with $p$ independent scheduling parameters in the form (1). The output feedback control law is considered for PID controller in the form

$$
u(t)=F(\theta) y+F_{d}(\theta) \dot{y}=F(\theta) C x+F_{d} C_{d} \dot{x}
$$

where

$$
F(\theta)=F_{0}+\sum_{i=1}^{p} F_{i} \theta_{i}
$$

is a static output feedback gain scheduled matrix for PI controller and

$$
F_{d}(\theta)=F_{d_{0}}+\sum_{i=1}^{p} F_{d_{i}} \theta_{i}
$$

is a static output feedback gain scheduled matrix for D part of controller. Substituting (2) to (1) and after

* Institute of Control and Industrial Informatics, Slovak University of Technology in Bratislava, Faculty of Electrical Engineering and Information Technology, Ilkovičova 3, 81219 Bratislava, Slovakia, adrian.ilka@stuba.sk, vojtech.vesely@stuba.sk 
some manipulation we can obtain the closed-loop system in the following form

$$
A_{d}(\theta) \dot{x}=A_{c}(\theta) x
$$

where

$$
\begin{aligned}
& A_{d}(\theta)=I-B(\theta) F_{d}(\theta) C_{d}, \\
& A_{c}(\theta)=A(\theta)+B(\theta) F(\theta) C .
\end{aligned}
$$

To access the performance quality a quadratic cost function [7] known from LQ theory is often used. In this note the original quadratic cost function is used, where weighting matrices depends on scheduling parameters. Using this approach we can affect on performance quality in each working point separately. The quadratic cost function is in the form

$$
J(\theta)=\int_{0}^{\infty}\left(x^{T} Q(\theta) x+u^{T} R u+\dot{x}^{T} S(\theta) \dot{x}\right) \mathrm{d} t
$$

where

$$
\begin{aligned}
Q(\theta) & =Q_{0}+\sum_{i=1}^{p} Q_{i} \theta_{i}, & Q_{i} & =Q_{i}^{T}>0, \\
S(\theta) & =S_{0}+\sum_{i=1}^{p} S_{i} \theta_{i}, & S_{i} & =S_{i}^{T}>0
\end{aligned}
$$

and $R>0$. The guaranteed cost is defined in a standard way.

Definition 1. Consider the system (1) with control algorithm (2). If there exists a control law $u^{*}$ and a positive scalar $J^{*}$ such that the closed-loop system (3) is stable and the value of closed-loop cost function (4) satisfies $J \leq J^{*}$ then $J^{*}$ is said to be a guaranteed cost and $u^{*}$ is said to be guaranteed cost control law for system (1).

Definition 2 [8]. The linear closed-loop system (3) for $\theta \in \Omega$ and $\dot{\theta} \in \Omega_{t}$ is affinally quadratically stable if and only if there exist $p+1$ symmetric matrices $P_{0}, P_{1}, \ldots, P_{p}$ such that

$$
P(\theta)=P_{0}+\sum_{i=1}^{p} P_{i} \theta_{i}>0
$$

and for the first derivative of Lyapunov function $V(\theta)=$ $x^{\top} P(\theta) x$ along the trajectory of closed-loop system (3) holds

$$
\frac{\mathrm{d} V(x, \theta)}{\mathrm{d} t}=x^{\top} V_{v}(\theta) x<0
$$

where

$$
\begin{aligned}
V_{v}(\theta) & =A_{c d}(\theta)^{\top} P(\theta)+P(\theta) A_{c d}(\theta)+\frac{\mathrm{d} P(\theta)}{\mathrm{d} t}, \\
\frac{\mathrm{d} P(\theta)}{\mathrm{d} t} & =\sum_{i=1}^{p} P_{i} \dot{\theta}_{i} \leq \sum_{i=1}^{p} P_{i} \rho_{i}, \\
A_{c d}(\theta) & =A_{d}(\theta)^{-1} A_{c}(\theta),
\end{aligned}
$$

assuming $P_{i}>0, i=1,2, \ldots p$.

From LQ theory we introduce the well known results.
Lemma 1. Consider the closed-loop system (3). Closedloop system (3) is affinally quadratically stable with guaranteed cost if and only if the following inequality holds

$$
B_{e}=\min _{u}\left\{\frac{d V(\theta)}{d t}+x^{\top} Q(\theta) x+u^{\top} R u+\dot{x}^{\top} S(\theta) \dot{x}\right\} \leq 0
$$

for all $\theta \in \Omega$ and $\dot{\theta} \in \Omega_{t}$

\section{MAIN RESULTS}

In this section we presented the gain scheduled controller design procedure which guarantees the affine quadratic stability and required guaranteed costs for all $\theta \in \Omega$ and $\dot{\theta} \in \Omega_{t}$. The main results for the case of gain scheduled closed-loop stability analysis reduces to LMI condition and for gain scheduled controller synthesis to BMI one.

The main results of this section is given by following theorem

Theorem 1. Closed-loop system (3) is AQS if there exists $p+1$ symmetric matrices $P_{0}, P_{1}, \ldots, P_{p}$, satisfying (5), matrices $N_{1}$ and $N_{2}$ and gain scheduled matrices $F(\theta)$ and $F_{d}(\theta)$ satisfying

$$
\begin{aligned}
& M_{i j}+M_{j i}<0, \\
& i, j=1,2, \ldots, p
\end{aligned}
$$

where

$$
M_{i j}=\left[\begin{array}{cc}
W_{11}^{i j} & W_{12}^{i j} \\
W_{12}^{i j} & W_{22}^{i j}
\end{array}\right]
$$

$$
W_{11}^{i j}=N_{1} A_{d}^{i j}+\left(A_{d}^{i j}\right)^{T} N_{1}+\frac{S_{0}}{\theta_{m}^{2}}+\frac{1}{\theta_{m}} S_{i}+C_{d}^{\top} F_{d}^{i j} C_{d},
$$

$$
W_{12}^{i j}=-N_{1} A_{c}^{i j}+\left(A_{d}^{i j}\right)^{\top} N_{2}^{\top}+\frac{P_{0}}{\theta_{m}^{2}}+\frac{1}{\theta_{m}} P_{i}+C_{d}^{\top} F_{d}^{i j} C,
$$$$
W_{22}^{i j}=-N_{2} A_{c}^{i j}-\left(A_{c}^{i j}\right)^{\top} N_{2}^{\top}+\frac{1}{\theta}_{m}^{2}\left(\sum_{k=1}^{p} P_{k} \rho_{k}+Q_{0}\right)
$$

$$
+\frac{1}{\theta_{m}} Q_{i}+C^{\top} F_{p}^{i j} C,
$$

$$
A_{d}^{i j}=\frac{1}{\theta_{m}^{2}} I-\left[\frac{1}{\theta_{m}^{2}} B_{0} F_{d_{0}}+\frac{1}{\theta_{m}} B_{0} F_{d_{i}}+\frac{1}{\theta_{m}} B_{i} F_{d_{0}}\right.
$$$$
\left.+B_{i} F_{d_{j}}\right] C_{d},
$$

$$
\begin{aligned}
F_{d}^{i j} & =\frac{1}{\theta_{m}^{2}} F_{d_{0}}^{\top} R F_{d_{0}}+\frac{1}{\theta_{m}}\left(F_{d_{0}} R F_{d_{i}}+F_{d_{i}}^{\top}+F_{d_{i}} R F_{d_{0}}\right) \\
& +F_{d_{i}}^{\top} R F_{d_{j}}, \\
\theta_{m} & =\sum_{i=1}^{p} \theta_{i}, \\
A_{c}^{i j} & =\frac{1}{\theta_{m}^{2}}\left(A_{0}+B_{0} F_{0} C\right)+\frac{1}{\theta_{m}}\left(A_{i}+B_{0} F_{i} C+B_{i} F_{0} C\right) \\
& +B_{i} F_{j} C,
\end{aligned}
$$




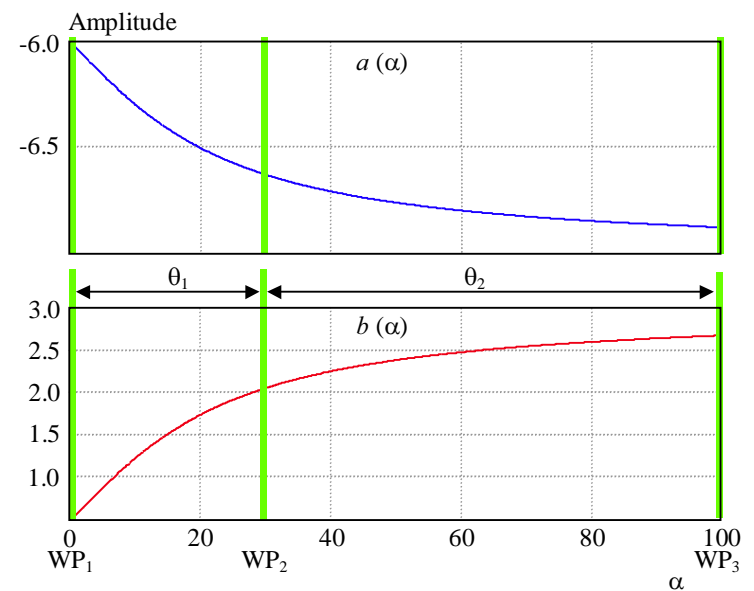

Fig. 1. Exogenous signal $\alpha(t)$

$$
\begin{aligned}
& F_{d_{r}}^{i j}=\frac{1}{\theta_{m}^{2}} F_{d_{0}}^{\top} R F_{0}+\frac{1}{\theta_{m}}\left(F_{d_{0}}^{\top} R F_{i}+F_{d_{i}} R F_{0}\right)+F_{d_{i}} R F_{j}, \\
& F_{p}^{i j}=\frac{1}{\theta_{m}^{2}} F_{0}^{\top} R F_{0}+\frac{1}{\theta_{m}}\left(F_{0}^{\top} R F_{i}+F_{i} R F_{0}\right)+F_{i} R F_{j} .
\end{aligned}
$$

Pr o of. Proof is based on Lemma 1. Time derivative of Lyapunov function using free matrix weighting approach is

$$
\frac{\mathrm{d} V}{\mathrm{~d} t}=z^{\top}\left[\begin{array}{ll}
Z_{11} & Z_{12} \\
Z_{21} & Z_{22}
\end{array}\right] z
$$

where

$$
\begin{aligned}
& Z_{11}=N_{1} A_{d}(\theta)+A_{d}^{\top}(\theta) N_{1}^{\top}, \\
& Z_{12}=-N_{1} A_{c}(\theta)+A_{d}^{\top}(\theta) N_{2}^{\top}+P(\theta), \\
& Z_{21}=-A_{c}^{\top}(\theta) N_{1}^{\top}+N_{2} A_{d}(\theta)+P(\theta), \\
& Z_{22}=-N_{2} A_{c}(\theta) A_{d}^{\top}(\theta) N_{2}^{\top}+\sum_{k=1}^{p} P_{k} \rho_{k},
\end{aligned}
$$

$N_{1}, N_{2} \in R^{n \times n}$ are auxiliary matrices.

When one substitutes to the third part of (7) control algorithm (2) and the obtained results with (3) to (7) after some manipulation we obtain (9). The proof is completed.

\section{EXAMPLE}

An illustrative example is taken from [9]. Consider a simple non-linear plant with parameter varying coefficients

$$
\begin{aligned}
& \dot{x}(t)=a(\alpha) x(t)+b(\alpha) u(t), \\
& y(t)=x(t)
\end{aligned}
$$

where $\alpha(t) \in R$ is an exogenous signal that changes the parameters of the plant as follows

$$
\begin{aligned}
& a(\alpha)=-6-\frac{2}{\pi} \arctan \frac{\alpha}{20}, \\
& b(\alpha)=\frac{1}{2}+\frac{5}{\pi} \arctan \frac{\alpha}{20} .
\end{aligned}
$$

Let the aim is to design gain-scheduled PID controller which will guarantee the closed-loop stability and guaranteed cost for $\alpha \in\langle 0,100\rangle$. We will demonstrate that with our gain-scheduled controller design we can obtain for closed-loop system practically identical behaviour for each working point. To be able to demonstrate this feature, let us divide the working area to 2 sections (with 3 working points) so that in each area where the plant parameter changes they are nearly linear (Fig. 1 - the green lines indicates the chosen working points).

In these working points calculated transfer functions are

$$
\begin{gathered}
\left.G_{s_{1}}\right|_{\alpha=0}=\frac{0.5}{s+6},\left.\quad G_{s_{2}}\right|_{\alpha=30}=\frac{2.064}{s+6.626} \\
\left.G_{s_{3}}\right|_{\alpha=100}=\frac{2.686}{s+6.874}
\end{gathered}
$$

We transform the above transfer functions into time domain to obtain scheduling model in the form (1). The obtained model was extended for gain scheduled PID controller design. The extended model is given as follows

$$
\begin{array}{rlrl}
A_{0} & =\left[\begin{array}{cc}
-6.4370 & 0 \\
1 & 0
\end{array}\right], & A_{1}=\left[\begin{array}{cc}
-0.3130 & 0 \\
0 & 0
\end{array}\right], \\
A_{2}=\left[\begin{array}{cc}
-0.1240 & 0 \\
0 & 0
\end{array}\right], & B_{0}=\left[\begin{array}{c}
1.5930 \\
0
\end{array}\right], \\
B_{1}=\left[\begin{array}{c}
0.7820 \\
0
\end{array}\right], & B_{2}=\left[\begin{array}{c}
0.3110 \\
0
\end{array}\right], \\
C=\left[\begin{array}{ll}
1 & 0 \\
0 & 1
\end{array}\right], & D=0 .
\end{array}
$$

Using Theorem 1 with weighting matrices $Q_{i}=q_{i} I$, $q_{1}=q_{2}=q_{3}=1 \times 10^{-4}, R=r I, r=1, S_{i}=s_{i} I$, $s_{1}=s_{2}=s_{3}=1 \times 10^{-7}$ we obtain gain scheduled controller in the form

$$
G_{r_{G S}}=G_{r_{0}}+G_{r_{1}} \theta_{1}+G_{r_{2}} \theta_{2}
$$

where

$$
\begin{aligned}
G_{r_{0}} & =\frac{0.3033 s^{2}+2.3036 s+2.0949}{s}, \\
G_{r_{1}} & =-\frac{3.93 \times 10^{-6} s^{2}+8.86 \times 10^{-5} s+3.13 \times 10^{-5}}{s}, \\
G_{r_{2}} & =-\frac{0.0724 s^{2}+1.6323 s+0.5773}{s} .
\end{aligned}
$$

Simulation results (Figs. 2, 3) confirm, that Theorem 1 holds, but we can see also that with equal $q_{i}, s_{i}$ we do not obtain identical behaviour in each working point. 


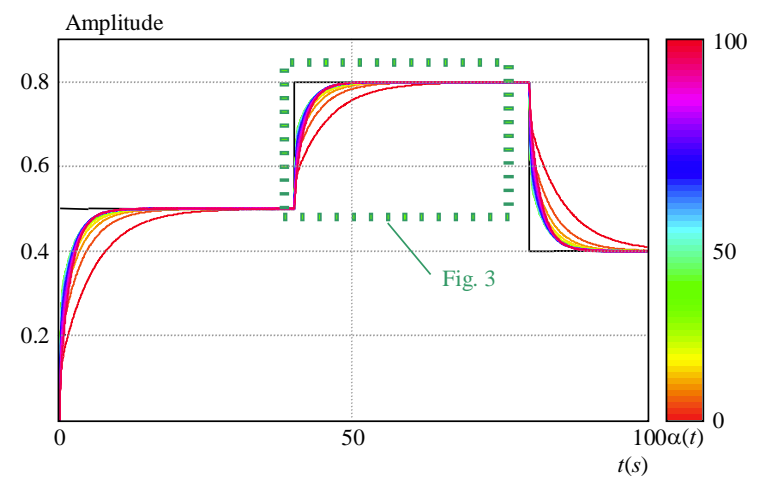

Fig. 2. Simulation results with GSC (15)

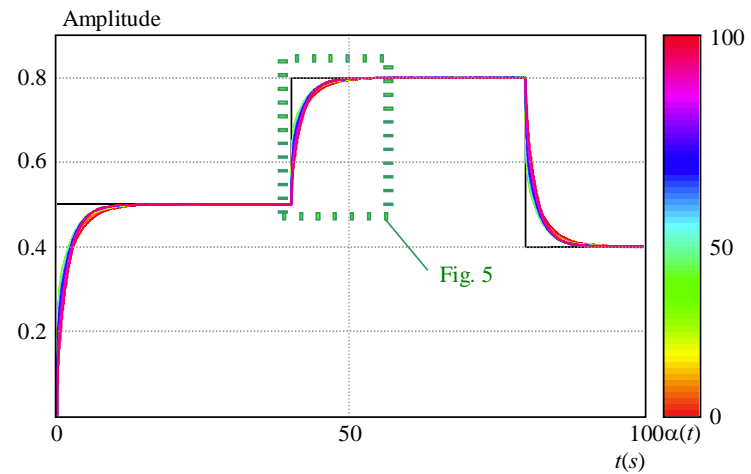

Fig. 4. Simulation results with GSC (16)

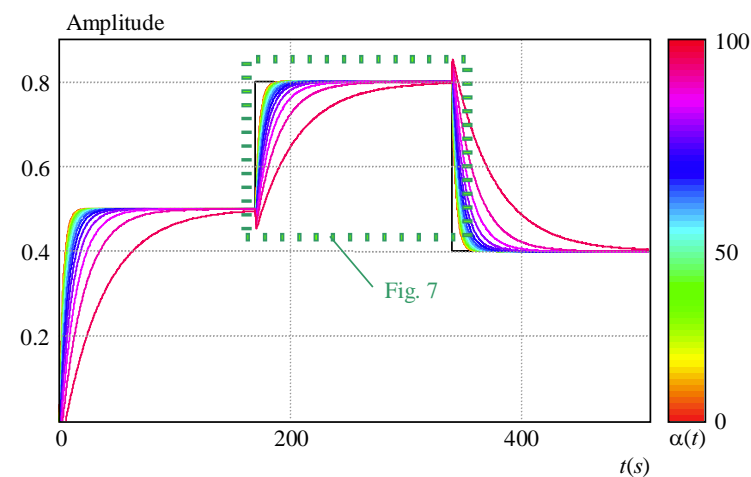

Fig. 6. Simulation results with GSC (17)
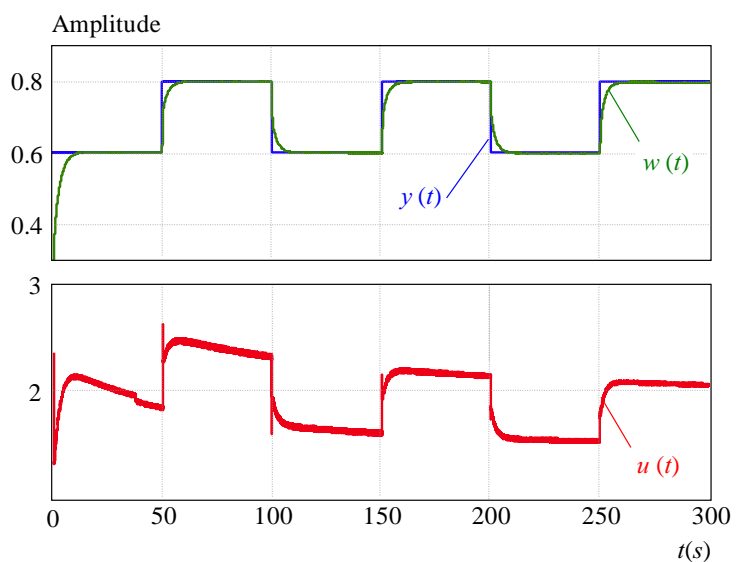

Fig. 8. Simulation results $(y(t), w(t), u(t))$ with GSC (16)

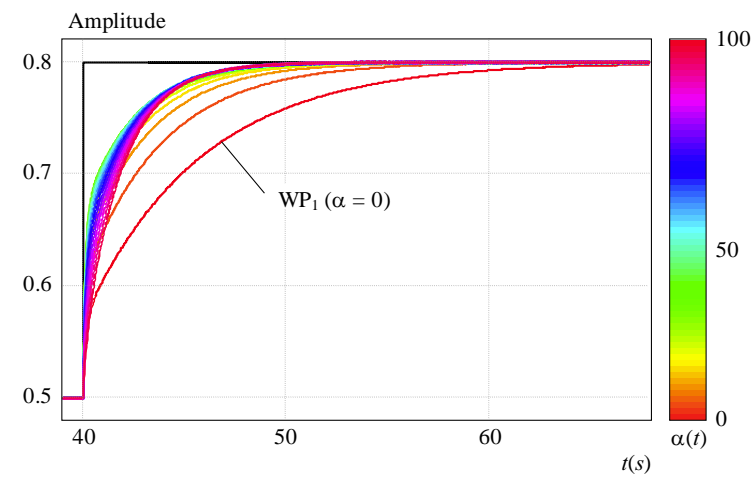

Fig. 3. Simulation results with GSC (15) - zoomed

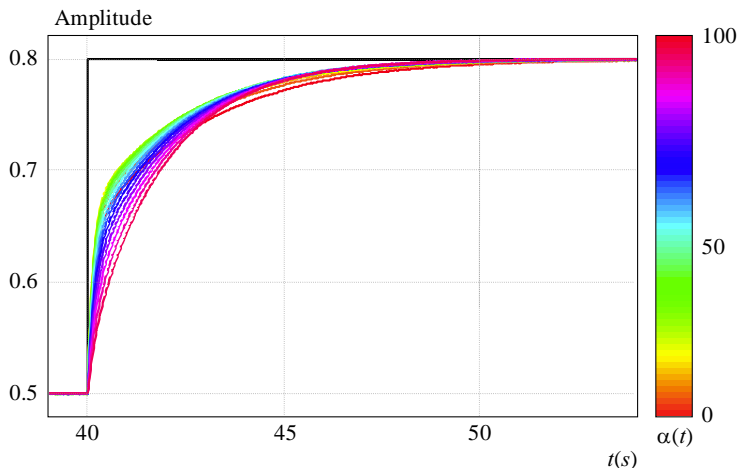

Fig. 5. Simulation results with GSC (16) - zoomed

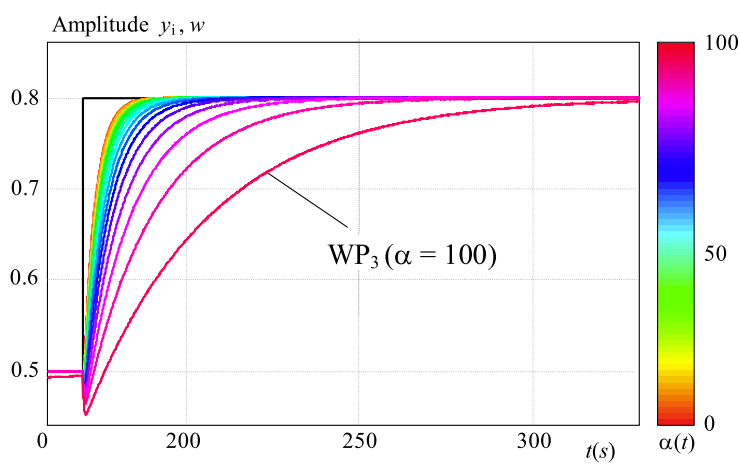

Fig. 7. Simulation results with GSC (17) - zoomed
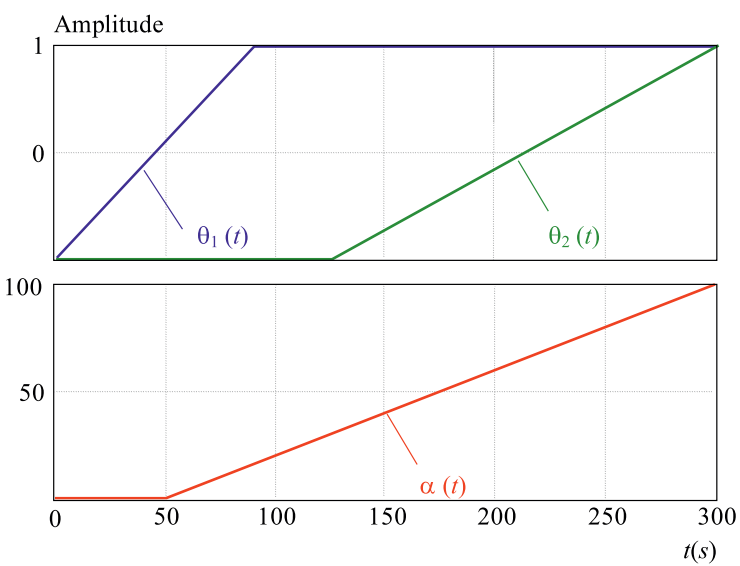

Fig. 9. Simulation results $(\theta(t), \alpha(t))$ with GSC (16) 
We can change the weighting matrices in the 1 . working point to get required performance quality. An another gain-scheduled controller was obtained with weighting matrices $Q_{i}=q_{i} I, q_{1}=1 \times 10^{-2}, q_{2}=q_{3}=1 \times 10^{-4}$, $R=r I, r=1, S_{i}=s_{i} I, s_{1}=s_{2}=s_{3}=1 \times 10^{-7}$,

$$
G_{r_{G S}}=G_{r_{0}}+G_{r_{1}} \theta_{1}+G_{r_{2}} \theta_{2}
$$

where

$$
\begin{aligned}
G_{r_{0}} & =\frac{0.5554 s^{2}+0.8513 s+2.7286}{s}, \\
G_{r_{1}} & =-\frac{0.0064 s^{2}+0.0559 s+0.0653}{s}, \\
G_{r_{2}} & =-\frac{0.0589 s^{2}+0.5173 s+0.6048}{s} .
\end{aligned}
$$

Simulation results (Figs. 4, 5) confirm, that with variable weighting matrices we can affect performance quality separately in each working points and we can tune the system to the desired conditions.

With our gain-scheduled controller design approach we can tune also the change of states with weighting matrices $S_{i}$ and we can influence to the overshot and oscillation and make the system more slowly.

Let the system to be more slowly in the last working point ( $\left.W P_{3}: \alpha=100\right)$. An another gain-scheduled controller was obtained with weighting matrices $Q_{i}=q_{i} I$, $q_{1}=1 \times 10^{-2}, q_{2}=q_{3}=1 \times 10^{-4}, R=r I, r=1$, $S_{i}=s_{i} I, s_{1}=s_{2}=1 \times 10^{-7}, s_{3}=1 \times 10^{-1}$

$$
G_{r_{G S}}=G_{r_{0}}+G_{r_{1}} \theta_{1}+G_{r_{2}} \theta_{2}
$$

where

$$
\begin{aligned}
G_{r_{0}} & =\frac{0.2161 s^{2}-0.1509 s+0.7893}{s}, \\
G_{r_{1}} & =-\frac{0.0095 s^{2}+0.1084 s+0.3770}{s}, \\
G_{r_{2}} & =-\frac{0.0088 s^{2}+0.1010 s+0.3515}{s} .
\end{aligned}
$$

Simulation results are shown in Figs. 6, 7. Gain-scheduled controller obtained with our controller design method is remains stable under slowly parameter changes too. This is shown in Figs. 8, 9 with gain-scheduled controller (16).

\section{CONCLUSION}

This paper addresses the problem to design gainscheduled controller which guarantee the closed-loop stability and performance for all scheduled parameter changes. The proposed original procedure is based on Lyapunov theory of stability, notion of guaranteed cost and BMI. Using original variable weighting matrices we can affect performance quality separately in each working points and we can tune the system to the desired condition through all parameter changes. Numerical example illustrate the effectiveness of the proposed approach.

\section{Acknowledgments}

The work has been supported by the Slovak Scientific Grant Agency, Grant No. 1/1241/12.

\section{REFERENCES}

[1] SATO, M. : Brief paper: Gain-Scheduled Output-Feedback Controllers Depending Solely on Scheduling Parameters via Parameter-Dependent Lyapunov Functions, Automatica 47 No. 12 (Dec 2011), 2786-2790.

[2] KÖROĞLU, H. : Robust Generalized Asymptotic Regulation via an LPV Controller without Parameter Derivative Dependence, 49th IEEE Conference on Decision and Control (CDC), Dec 2010, pp. 4965-4971.

[3] FAN WANG-BALAKRISHNAN, V.: Improved Stability Analysis and Gain-Scheduled Controller Synthesis for Parameter-Dependent Systems, IEEE Transactions on Automatic Control 47 No. 5 (May 2002), 720-734.

[4] LEITH, D. J.-LEITHEAD, W. E. : Survey of Gain-Scheduling Analysis and Design, International Journal of Control 73 No. 11 (2000), 1001-1025.

[5] RUGH, W. J.-SHAMMA, J. S. : A Survey of Research on Gain-Scheduling, Automatica 36 (Sep 2000), 1401-1425.

[6] Gahinet, P.-APKARIAN, P.-CHILALI, M. : Affine Parameter-Dependent Lyapunov Functions and Real Parametric Uncertainty, IEEE Transactions on Automatic Control 41 No. 3 (Mar 1996), 436-442.

[7] ENGWERDA, J.-WEEREN, A. : A Result on Output Feedback Linear Quadratic Control, Automatica 44 No. 1 (2008), 265-271.

[8] APKARIAN, P.-GAHINET, P.-BECKER, G. : Self-Scheduled $H_{\infty}$ Control of Linear Parameter-Varying Systems: A Design Example, Automatica 31 No. 9 (1995), 1251-1261.

[9] STEWART, G. E.: A Pragmatic Approach to Robust Gain Scheduling, 7th IFAC Symposium on Robust Control Design, vol. 7, Aalborg Congress \& Culture Centre, Denmark, 2012, pp. 355-362.

Received 11 July 2013

Adrian Ilka (Ing) was born in Dunajská Streda, Slovakia in 1987. He received Bachelor of Science degree from the Faculty of Electrical Engineering and Information Technology, Slovak University of Technology in Bratislava in 2010 and Master of Science degree in technical cybernetics in 2012 at the same place where he is from 2012 until now doing further studies to get his $\mathrm{PhD}$. title. He is interested in power system control, robust control, Lyapunov theory of stability, linear matrix inequalities and gain-scheduled control.

Vojtech Veselý (Prof, Ing, DrSc) was born in Veĺké Kapušany, Slovakia, in 1940. He received an MSc in Electrical Engineering from the Leningrad Electrical Engineering Institute, St. Peterburg, Russia, in 1964, the PhD and the DSc degrees from the Slovak University of Technology, Bratislava, Slovak Republic, in 1971 and 1985, respectively. Since 1964 he has been with the Department of Automatic Control Systems, STU FEI in Bratislava. Since 1986 he has been a full professor. His research interests include the areas of power system control, decentralised control of large-scale systems, robust control, predictive control and optimisation. He is author or coauthor of more than 250 scientific papers. 